\title{
Mathematical Modeling of Porous Medium for Sound Absorption Simulations: Application of Multi-Scales and Homogenization
}

\author{
Allen Teagle-Hernandez ${ }^{1,2}$, Ortwin Ohtmer ${ }^{1}$, Duyen Nguyen ${ }^{1}$ \\ ${ }^{1}$ Mecahnical and Aerospace Engineering Department, California State University, Long Beach, USA \\ ${ }^{2}$ Vibro-Acoustic Department, HSEA Tech. Inc., Nagoya, Japan \\ Email: allen.teagle@csulb.edu
}

How to cite this paper: Teagle-Hernandez, A., Ohtmer, O. and Nguyen, D. (2018) Mathematical Modeling of Porous Medium for Sound Absorption Simulations: Application of Multi-Scales and Homogenization. Journal of Applied Mathematics and Physics, 6, 2705-2717.

https://doi.org/10.4236/jamp.2018.612224

Received: December 5, 2018

Accepted: December 28, 2018

Published: December 31, 2018

\begin{abstract}
The modeling of porous medium has many applications whose techniques can be used in the fields of automotive, aerospace, oil exploration, and biomedical. This work concentrates on the Noise and Vibration (NV) development of automotive interiors but the ideas can be translated to the aforementioned areas. The NV development requires the setting of NV targets at different levels. These targets are then translated to TL (Transmission Loss), IL (Insertion Loss), and Alpha (absorption) performance. Therefore, the ability to manage an efficient product development cycle, that entails analyzing vibro-acoustic environments, hinges on the premise that accurate TL, IL, or Alpha values pertaining to the different multi-layered porous materials can be calculated. Thus, there is a need to have a thorough understanding of the physics behind the energy dissipating mechanism that includes the effects of the fluid meandering through the pores of the material. The goal of this series is to model the acoustic and dynamic coupling via multi-scale and homogenizations techniques, thus subsequently understand where to incorporate the concepts of dynamic tortuosity, viscous and thermal permeability, as well as viscous and thermal lengths. This study will allow the ability to get a better understanding of the underlying processes and also provides tools to create practical concepts for determining the coefficients of the macroscopic equations. This will assist in attaining novel ideas for NV absorption and insulation.
\end{abstract}

\section{Keywords}

Porous Medium, Multi-Scale, Homogenization, Fluid Structure Interaction 


\section{Introduction}

$\mathrm{NV}$ development begins by assigning NV targets for different systems. Using an automobile development cycle as an example, this translates to assigning NV targets to systems like dashboard, floor, roof, trunk, and other systems of the automobile. These targets dictate TL, IL and Alpha performances that are projected to different parts that the system comprises of. These performance levels are usually derived via a Hybrid Statistical Energy Analysis (HSEA) technique [1] [2] [3]. The aggregate sound attenuation performance of each component is affected by the multi-layer porous materials that are utilized to manufacture it. NV specialists run simulations in order to decide the optimum combination.

Material suppliers furnish layer parameters such as porosity, tortuosity, resistivity, foam bulk modulus, skeleton Young's modulus, viscous and thermal lengths that are then applied to calculate flat sample TL, IL, and Alpha. Quite often, OEM engineers plug in values pertaining to these parameters, but have little understanding of the energy absorbing or sound blocking mechanism. Decisions are based on past experiences. The difficulty is to find a work that encompasses the gamut of equations required and clearly explains the physics behind transmission and absorption of energy. This work builds a bridge between the gaps in order to obtain a more fundamental understanding. In this part of the series the goal is to derive the coupled fluid/structural equations. The macro-scale equations are obtained by applying the multi scales and homogenization techniques. The scales of the pores are small compared to the macroscales. The process will show how energy is being dissipated due to fluid/structure interaction; the dilatational/compressional interplay between the acoustic/fluid and structural medium.

The encapsulation of the acoustic medium due to how tortuous the foam material is and the mass entrapped in the viscous boundary layer are incorporated in the above equations. There is also a boundary layer where the flow is not adiabatic, therefore a loss/gain of energy due to thermal exchange will occur. This thermal exchange also changes the acoustic bulk modulus at certain frequencies and in turn changing the speed of sound. This paper ties the aforementioned physical phenomena to the parameters of tortuosity, dynamic viscous and thermal permeability, viscous as well as thermal lengths. The derivation of the equation bands together ideas and techniques presented in [4] [5] [6] [7]. Part 2 of this series details how to incorporate the foam equations with other type of layers, e.g. panels, air gap, other foams, via the Transfer Matrix Method (TMM) and Finite Transfer Matrix Method (FTMM) in order to calculate TL, IL, and Alpha.

\section{Formulation}

\subsection{Basic Tensor Calculus and Notation}

This body of work takes advantage of tensor notation and tensor calculus. This notational generalization makes it easy to describe the physics regardless of the 
abstract methodology used to define the dynamics of the system. In order to clearly define ideas, the Einstein summation convention is used in which covariant index followed by the identical contravariant index is implicitly summed over. Variables written in bold are tensors of rank 1, i.e. vectors. In the meantime, a bold letter with a special tilde as shown here, $\vec{A}$, is a tensor of rank 2 . The covariant derivative of a contravariant vector, $\boldsymbol{A}^{i}$ is given as

$$
\nabla_{j} A^{i}=\frac{\partial A^{i}}{\partial x^{j}}+\Gamma_{j k}^{i} A^{k}
$$

Additionally, the use of the divergence of a second rank tensor, $\ddot{\boldsymbol{T}}^{i j}$, is defined as

$$
\nabla_{i} \ddot{\boldsymbol{T}}^{i j}=\partial_{i} \ddot{\boldsymbol{T}}^{i j}+\Gamma_{i k}^{i} \ddot{\boldsymbol{T}}^{k j}+\Gamma_{i k}^{i} \ddot{\boldsymbol{T}}^{i k}
$$

In Equation (1) and (2), $\Gamma_{i k}^{i}$, is the Chritoffel symbol of the second kind.

\subsection{Fluid-Structure Interaction: Two-Scale Expansion}

The pore composition of the porous medium has amicroscopic length $l$, while the macroscopic length is designated $L$. The material is statistically homogenous at the macroscopic scale. The ratio of the two length scale is set as $\epsilon=\frac{l}{L}$ where $\epsilon \ll 1$. The different acoustic and vibration variables will depend on two scales: a slow scale $\boldsymbol{x}$ and a fast scale $\boldsymbol{y}=\frac{\boldsymbol{x}}{\epsilon}$. The two-scale expansion is applied to the coupled fluid-structure equations. For relatively small viscosity values, conditions found in acoustics, the intrinsic viscous relaxation time is $\frac{l^{2}}{v}$, here $v$ is the kinematic viscosity $\frac{\eta}{\rho_{f}}$. In order to correctly compare orders of asymptotic expansion the dimensionless scaled terms of the equations of motion are derived and hence the right scaling factors are required. Due to the dynamic viscous permeability conditions, acoustic pressure and structural stresses are scaled with respect to $\tilde{p}=\frac{L \eta^{2}}{\rho_{o} l^{3}}$. Concurrently, due to the dynamic thermal permeability conditions the temperature fluctuation is scaled with respect to $\tilde{T}=\frac{L \eta^{3}}{\rho_{o}^{2} l^{3} \kappa}$. The dimensionless form of the coupled Navier-Stokes and structural equations along with the fluid/structure boundary conditions are given as

$$
\begin{gathered}
-i \omega^{*} \boldsymbol{v}^{*}=-\frac{1}{\epsilon} \boldsymbol{\nabla}^{*} \boldsymbol{p}^{*}+\nabla^{* 2} \boldsymbol{v}^{*}+\left(\frac{1}{3}+\frac{\xi}{\eta}\right) \nabla^{*}\left(\boldsymbol{\nabla}^{*} \cdot \boldsymbol{v}^{*}\right) \text { in } D_{f} \\
-i \omega^{*}\left(p^{*}-\frac{\gamma-1}{\gamma} \operatorname{Pr} T^{*}\right)=-\frac{1}{\gamma \varepsilon} \nabla^{*} \cdot \boldsymbol{v}^{*} \text { in } D_{f} \\
-i \omega^{*} \operatorname{Pr} T^{*}=-i \omega^{*} p^{*}+\nabla^{* 2} T \text { in } D_{f} \\
\boldsymbol{v}^{*}=-i \omega^{*} \boldsymbol{u}^{*} \text { in } \partial D_{f}=\partial D_{s} \\
\boldsymbol{n} \cdot \overrightarrow{\boldsymbol{\sigma}^{*}}=\boldsymbol{n} \cdot \boldsymbol{\tau}^{*} \text { in } \partial D_{f}=\partial D_{s}
\end{gathered}
$$




$$
\begin{gathered}
-\omega^{* 2} \boldsymbol{u}^{*}=\frac{\boldsymbol{\rho}_{f}}{\boldsymbol{\rho}_{s}} \frac{1}{\epsilon} \nabla^{*} \overleftrightarrow{\tau}^{*} \text { in } D_{s} \\
\overleftrightarrow{\boldsymbol{\tau}^{*}}=\overleftrightarrow{\boldsymbol{C}^{* i j k l}} \frac{\boldsymbol{\nabla}}{\epsilon} \boldsymbol{u}^{*} \text { in } D_{s}
\end{gathered}
$$

The dimensionless/scaled version of each variable is denoted with an asterisk. For each of the variables in Equations (3)-(9) are represented by a two scale expansion $f=f_{o}(x, y)+\epsilon f_{1}(x, y)+O\left(\epsilon^{2}\right)$. The covariant derivative becomes $\nabla^{*}=\nabla_{y}^{*}+\epsilon \nabla_{x}^{*}$. Applying the expansion and collecting all terms of $\mathrm{O}(1 / \square)$ the following are obtained

$$
\begin{aligned}
& \nabla_{y}^{*} p_{o}=0 \\
& -\frac{1}{\gamma} \nabla_{y}^{*} \cdot \boldsymbol{v}_{o}^{*}=\mathbf{0} \\
& \nabla_{y}^{*} \cdot \tau_{o}^{*}=\mathbf{0} \\
& \overleftrightarrow{\boldsymbol{C}^{* i j k l}} \nabla_{y}^{*} \boldsymbol{u}_{o}^{*}=\mathbf{0}
\end{aligned}
$$

Collecting all $O(1)$ terms the following key equations are obtained

$$
\begin{gathered}
-i \omega^{*} \boldsymbol{v}_{o}^{*}=-\nabla_{y}^{*} p_{1}^{*}-\nabla_{x}^{*} p_{o}^{*}+\nabla_{y}^{* 2} \boldsymbol{v}_{o}^{*} \text { in } D_{f} \\
-i \omega^{*}\left(p_{o}^{*}-\frac{\gamma-1}{\gamma} \operatorname{Pr} T_{o}^{*}\right)=-\frac{1}{\gamma} \nabla_{y}^{*} \cdot \boldsymbol{v}_{1}^{*}-\frac{1}{\gamma} \nabla_{x}^{*} \cdot \boldsymbol{v}_{o}^{*} \text { in } D_{f} \\
-i \omega^{*} \operatorname{Pr} T_{o}^{*}=-i \omega^{*} p_{o}^{*}+\nabla_{y}^{* 2} T_{o}^{*} \text { in } D_{f} \\
-\omega^{* 2} \boldsymbol{u}_{o}^{*}=\frac{\rho_{f}}{\rho_{s}}\left(\nabla_{y}^{*} \cdot \overleftrightarrow{\tau_{1}^{*}}+\nabla_{x}^{*} \cdot \overleftrightarrow{\tau_{o}^{*}}\right) \text { in } D_{s} \\
\overleftrightarrow{\tau_{o}^{*}}=\overleftrightarrow{\boldsymbol{C}^{* i j k l}} \nabla_{y}^{*} \boldsymbol{u}_{1}^{*}+\overleftrightarrow{\boldsymbol{C}^{* i j k l} \nabla_{x}^{*} \boldsymbol{u}_{o}^{*} \text { in } D_{s}}
\end{gathered}
$$

To better explain the interaction conditions the relative displacement $\boldsymbol{w}(\boldsymbol{x}, \boldsymbol{y})$ is introduced,

$$
\boldsymbol{v}_{o}^{*}=-i \omega^{*}\left[\boldsymbol{u}_{o}^{*}(x)+\boldsymbol{w}^{*}(\boldsymbol{x}, \boldsymbol{y})\right] \text { in } D_{f}
$$

and is incorporated into Equations (14) and (15)

$$
\begin{gathered}
-\omega^{* 2} \boldsymbol{u}_{o}^{*}(\boldsymbol{x})-\omega^{* 2} \boldsymbol{w}^{*}(\boldsymbol{x}, \boldsymbol{y})=-\nabla_{y}^{*} p_{1}^{*}-\nabla_{x}^{*} p_{o}^{*}-\nabla_{y}^{* 2} \boldsymbol{w}^{*}(\boldsymbol{x}, \boldsymbol{y}) \text { in } D_{f} \\
-i \omega^{*}\left(p_{o}^{*}-\frac{\gamma-1}{\gamma} \operatorname{Pr} T_{o}^{*}\right) \\
=-\frac{1}{\gamma} \nabla_{y}^{*} \cdot \boldsymbol{v}_{1}^{*}+i \omega^{*} \frac{1}{\gamma} \nabla_{x}^{*} \cdot \boldsymbol{u}_{o}^{*}+i \omega^{*} \frac{1}{\gamma} \nabla_{x}^{*} \cdot \boldsymbol{w}^{*}(\boldsymbol{x}, \boldsymbol{y}) \text { in } D_{f} \\
-i \omega^{*} \operatorname{Pr} T_{o}^{*}=-i \omega^{*} p_{o}^{*}+\nabla_{y}^{* 2} T_{o}^{*} \text { in } D_{f}
\end{gathered}
$$

Thus, the new boundary condition becomes $\boldsymbol{w}(\boldsymbol{x}, \boldsymbol{y})=\mathbf{0}$ at $\partial D_{f}=\partial D_{s}$

In the meantime, to solve the coupled partial differential Equations (20)-(22), it is assumed that there exist a rank 1 operator, $\boldsymbol{P}^{*}(\boldsymbol{x}, \boldsymbol{y})$, a second rank tensor $\overleftrightarrow{\boldsymbol{V}^{*}}(\boldsymbol{x}, \boldsymbol{y})$ and a scalar $\Theta(x, y)$ that act as transfer functions or operators that satisfy the following 


$$
\begin{gathered}
p_{1}^{*}=P^{*}(\boldsymbol{x}, \boldsymbol{y})\left[-\nabla_{x}^{*} p_{o}^{*}-\omega^{* 2} \boldsymbol{u}_{o}^{*}(\boldsymbol{x})\right] \\
-i \omega^{*} \boldsymbol{w}^{*}(\boldsymbol{x}, \boldsymbol{y})=\overleftrightarrow{\boldsymbol{V}^{*}}\left[-\nabla_{x}^{*} p_{o}^{*}-\omega^{* 2} \boldsymbol{u}_{o}^{*}(\boldsymbol{x})\right] \\
T_{o}^{*}=\Theta(\boldsymbol{x}, \boldsymbol{y})\left(-i \omega^{*} p_{o}^{*}\right)
\end{gathered}
$$

In [8] [9], a more detailed analysis at the microscopic level is constructed. There, it is shown that the microscopic dynamics produces exponential decaying effects on the transfer functions $\overrightarrow{\boldsymbol{V}^{*}}(\boldsymbol{x}, \boldsymbol{y})$ and $\Theta(x, y)$. Fluid equations pertaining to the viscous boundary layer relates this decaying effects on viscous length. For the thermal counterpart, the decaying effects due to the entropy boundary layer are related to thermal length. Homogeneity is obtained by averaging at the microscopic level, i.e. with respect to $y$, thus the following relation is obtained

$$
-i \omega \overline{\boldsymbol{w}(\boldsymbol{x})}=\overline{\overline{\boldsymbol{V}^{*}}} l^{2} \varphi\left[-\nabla_{x} p_{o}-\rho_{o} \omega^{2} \boldsymbol{u}_{o}(\boldsymbol{x})\right]
$$

Comparing this equation and the definition of dynamic permeability will determine that

$$
\boldsymbol{k}(\omega)=\overline{\overline{\boldsymbol{V}^{*}}} l^{2} \varphi
$$

Therefore

$$
-i \omega \overline{\boldsymbol{w}(\boldsymbol{x})}=\frac{\overrightarrow{\boldsymbol{k}}(\omega)}{\eta}\left[-\nabla_{x} p_{o}-\rho_{o} \omega^{2} \boldsymbol{u}_{o}(\boldsymbol{x})\right]
$$

Taking the inverse of $\boldsymbol{k}(\omega)$, Equation (27) can be rearranged as

$$
\begin{aligned}
& \frac{i \eta}{\boldsymbol{k}(\omega) \omega \rho_{f}} \rho_{f} \frac{\boldsymbol{d}^{2} \overline{\boldsymbol{w}(\boldsymbol{x})}}{\boldsymbol{d} \boldsymbol{t}^{2}}=-\nabla_{x} p_{o}-\rho_{o} \omega^{2} \boldsymbol{u}_{\boldsymbol{o}}(\boldsymbol{x}) \\
& =\alpha(\omega) \rho_{f} \frac{\boldsymbol{d}^{2} \overline{\boldsymbol{w}(\boldsymbol{x})}}{\boldsymbol{d \boldsymbol { t } ^ { 2 }}}=-\nabla_{x} p_{o}-\rho_{o} \omega^{2} \boldsymbol{u}_{\boldsymbol{o}}(\boldsymbol{x}) \alpha(\omega)=\frac{i \eta}{\boldsymbol{k}(\omega) \omega \rho_{f}}
\end{aligned}
$$

$\alpha(\omega)$ is known as Dynamic tortuosity. Variables with an overbar, e.g. $\overline{\boldsymbol{w}(\boldsymbol{x})}$, are ones that have been averaged at the microscopic level.

The dimensional form of Equation (25) is

$$
T_{o}=l^{2} \Theta(x, y)\left(-i \omega p_{o}\right)
$$

By taking the microscopic average of the above equation one will obtain

$$
T_{o}=k^{\prime}\left(-i \omega p_{o}\right) \text { such that } k^{\prime}=l^{2} \overline{\Theta(x, y)}
$$

Replacing the temperature with this relationship to pressure in Equation (21), the following is obtained

$$
\begin{aligned}
& -i \omega^{*} p_{\mathrm{o}}^{*}\left(\gamma+(\gamma-1) P r k^{*^{*}} i \omega^{*}\right) \\
& =-\nabla_{y}^{*} \cdot \boldsymbol{v}_{1}^{*}+i \omega^{*} \nabla_{x}^{*} \cdot \boldsymbol{u}_{o}^{*}+i \omega^{*} \nabla_{x}^{*} \cdot \boldsymbol{w}^{*}(\boldsymbol{x}, \boldsymbol{y}) \text { in } \mathrm{D}_{\mathrm{f}}
\end{aligned}
$$

The term $\beta_{a}^{*}=\gamma+(\gamma-1) \operatorname{Pr}^{\prime *} i \omega^{*}$ is the compressibility factor in dimensionless form. Set $\kappa_{a}^{*}=\frac{1}{\beta_{a}^{*}}$ (bulk modulus for the acoustic medium) and ap- 
ply this in the last equation above

$$
p_{o}^{*}-\frac{\kappa_{a}^{*}}{i \omega^{*}} \nabla_{y}^{*} \cdot \boldsymbol{v}_{1}^{*}+\kappa_{a}^{*} \nabla_{x}^{*} \cdot \boldsymbol{u}_{o}^{*}+\kappa_{a}^{*} \nabla_{x}^{*} \cdot \boldsymbol{w}^{*}(\boldsymbol{x}, \boldsymbol{y})=0 \text { in } D_{f}
$$

After taking the microscopic average of (31), the following expression is obtained

$$
\varphi p_{o}^{*}-\overline{\frac{\kappa_{a}^{*}}{i \omega^{*}} \nabla_{y}^{*} \cdot v_{1}^{*}}+\kappa_{a}^{*} \varphi \nabla_{x}^{*} \cdot \boldsymbol{u}_{o}^{*}+\overline{\kappa_{a}^{*} \nabla_{x}^{*} \cdot \boldsymbol{w}^{*}(\boldsymbol{x}, \boldsymbol{y})}=0 \text { in } D_{f}
$$

\subsection{Structural: Finite Element - Representative Volume Element}

The structural skeleton of the porous medium will be split up into identical unit cells where the macroscopic variables will represent conditions at the boundary, while microscopic variables will be tied to conditions interior of the unit cell. The boundary conditions and structural characteristics of the unit cell will be assumed statistically periodic. Special boundary conditions and interior forces are applied in order to obtain a set of Basis functions that will expand the interior solutions. A set of equations that will relate boundary stresses to interior strains will be easily obtained by representing

$$
\overrightarrow{\tau_{o}^{*}}=\overleftrightarrow{\boldsymbol{C}^{* i j k l}} \nabla_{y}^{*} \boldsymbol{u}_{1}^{*}+\overleftrightarrow{\boldsymbol{C}^{* i j k l}} \nabla_{x}^{*} \boldsymbol{u}_{o}^{*} \text { in } D_{s}
$$

In order to obtain a relationship between macroscopic strain and macroscopic stresses and considering the impedance from the micro-scale portion the following finite element form is used

$$
\left[\begin{array}{ll}
C_{i i}^{*} & C_{i b}^{*} \\
C_{b i}^{*} & C_{b b}^{*}
\end{array}\right]\left\{\begin{array}{l}
\nabla_{y}^{*} \boldsymbol{u}_{1}^{*} \\
\nabla_{x}^{*} \boldsymbol{u}_{o}^{*}
\end{array}\right\}=\left\{\begin{array}{c}
0 \\
\overrightarrow{\tau^{*}}
\end{array}\right\}
$$

$C_{i i}^{*}$ are the elements pertaining to interior nodes, $C_{i b}^{*}$ and $C_{b i}^{*}$ are the coupling elements, while $C_{b b}^{*}$ are the elements pertaining to the boundary nodes. The assumption that there exist a potential energy for the fluid-structure problem forces $C_{i b}^{*}$ and $C_{b i}^{*}$ to be transpose operators from each other. From the top part of the above matrix formulation an expression for $\nabla_{y}^{*} \boldsymbol{u}_{1}^{*}$ as a function of $\nabla_{x}^{*} \boldsymbol{u}_{o}^{*}$ can be obtained

$$
\nabla_{y}^{*} \boldsymbol{u}_{1}^{*}=-\left[C_{i i}^{*}\right]^{-1} C_{i b}^{*} \nabla_{x}^{*} \boldsymbol{u}_{o}^{*}
$$

thus

$$
\overrightarrow{\boldsymbol{\tau}}^{*}=\left[-C_{b i}^{*}\left[C_{i i}^{*}\right]^{-1} C_{i b}^{*}+C_{b b}^{*}\right] \nabla_{x}^{*} \boldsymbol{u}_{o}^{*}
$$

The interior basis functions can be obtained by solving the following matrix formulation

$$
\left[\begin{array}{cc}
C_{i i}^{*} & C_{i b}^{*} \\
C_{b i}^{*} & C_{b b}^{*}
\end{array}\right]\left\{\begin{array}{l}
\nabla_{y}^{*} \boldsymbol{u}_{1}^{*} \\
\nabla_{x}^{*} \boldsymbol{u}_{o}^{*}
\end{array}\right\}=\left[\begin{array}{cc}
C_{i i}^{*} & C_{i b}^{*} \\
C_{b i}^{*} & C_{b b}^{*}
\end{array}\right]\left\{\begin{array}{c}
\nabla_{y}^{*} \boldsymbol{u}_{1}^{*} \\
0
\end{array}\right\}=\left\{\begin{array}{c}
\overrightarrow{\boldsymbol{I}}_{p} p_{0}^{*} \\
\overrightarrow{\boldsymbol{\tau}_{i}^{*}}
\end{array}\right\}
$$

where $\overrightarrow{\boldsymbol{I}}_{p}$ is a special tensor, of rank 2, that transforms the scalar, $p_{o}$, pressure into its tensor form and applies it normal to the structural surface. It should be 
clear that $C_{i i}^{*}, C_{i b}^{*}, C_{b i}^{*}$, and $C_{b b}^{*}$ are $4^{\text {th }}$ order contravariant tensors. The stress component that is transferred to the boundary nodes will be equal to

$$
\overrightarrow{\tau_{i}^{*}}=C_{b i}^{*}\left[C_{i i}^{*}\right]^{-1} \overleftrightarrow{\boldsymbol{I}_{p}} p_{o}^{*}
$$

By combining the two concepts the equations for microscopic strains and macroscopic stress is

$$
\begin{gathered}
\nabla_{y}^{*} \cdot u_{1}^{*}=\operatorname{tr}\left[C_{i i}^{*}\right]^{-1} \ddot{\boldsymbol{I}}_{o}^{*}-\operatorname{tr}\left[C_{i i}^{*}\right]^{-1} C_{i b}^{*} \nabla_{x}^{*} \boldsymbol{u}_{o}^{*} \\
\overrightarrow{\boldsymbol{\tau}_{o}}=\left[-C_{b i}\left[C_{i i}\right]^{-1} C_{i b}-C_{b b}\right] \nabla_{x} \boldsymbol{u}_{o}+C_{b i}\left[C_{i i}\right]^{-1} \overrightarrow{\boldsymbol{I}}_{p} p_{o}
\end{gathered}
$$

From the acoustic relations, Equation (20) can be placed into the following form

$$
-\omega^{* 2} \boldsymbol{u}_{o}^{*}(x)-\omega^{* 2} \boldsymbol{w}^{*}(\boldsymbol{x}, \boldsymbol{y})+\nabla_{x}^{*} p_{o}^{*}=\nabla_{y}^{*} \cdot \overrightarrow{\boldsymbol{\sigma}}_{1}^{*} \text { in } D_{f}
$$

Taking the microscopic average of this equation and utilizing Green's theorem Equation (41) becomes

$$
\begin{aligned}
& \omega^{* 2} \varphi \boldsymbol{u}_{o}^{*}(\boldsymbol{x})+\omega^{* 2} \overline{\boldsymbol{w}^{*}(\boldsymbol{x}, \boldsymbol{y})}-\varphi \nabla_{x}^{*} p_{o}^{*} \\
& =-\lim _{R \rightarrow \infty} \frac{1}{\frac{4}{3} \pi R^{3}} \iint_{\text {boundary }} \overrightarrow{\boldsymbol{\sigma}_{1}^{*}} \cdot \boldsymbol{d} \boldsymbol{A}_{y} \text { in } D_{f}
\end{aligned}
$$

Similarly, the same procedure is done to the structural Equation, (17)

$$
\omega^{* 2} \frac{\overline{\boldsymbol{\rho}_{s}}}{\overline{\boldsymbol{\rho}_{f}}} \varphi \boldsymbol{u}_{o}^{*}+\overline{\nabla_{x}^{*} \cdot \overline{\tau_{o}^{*}}}=-\lim _{R \rightarrow \infty} \frac{1}{\frac{4}{3} \pi R^{3}} \iint_{\text {boundary }} \overrightarrow{\tau_{1}^{*}} \cdot \boldsymbol{d} \boldsymbol{A}_{y} \text { in } D_{s}
$$

Due to the boundary conditions at the interface, Equation (7), the foregoing equations becomes

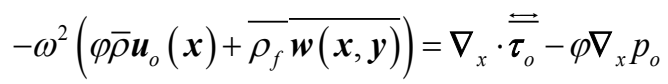

where $\bar{\rho}=\overline{\rho_{s}}+\overline{\rho_{f}}$.

\subsection{Coupled Equations}

Equation (28) will be rewritten as

$$
-\omega^{2}\left(\rho_{f} \boldsymbol{u}_{o}(\boldsymbol{x})+\alpha(\omega) \rho_{f} \overline{\boldsymbol{w}(\boldsymbol{x}, \boldsymbol{y})}\right)=-\nabla_{x} p_{o}
$$

Reintroducing dimensions to the compressibility factor $\beta_{a}{ }^{*}$ one will get

$$
\beta_{a}=\gamma+(\gamma-1) \frac{P r}{v} k^{\prime} i \omega
$$

The final dimensional form pertaining to the acoustic is

$$
\varphi p_{o}+\kappa_{a} \nabla_{y} \cdot \overline{\boldsymbol{u}_{1}}+\kappa_{a} \varphi \nabla_{x} \cdot \overline{\boldsymbol{u}_{o}}+\kappa_{a} \nabla_{x} \cdot \overline{\boldsymbol{w}(\boldsymbol{x}, \boldsymbol{y})}=0 \text { in } D_{f}
$$

Plugging in Equation (39) into (47), the dimensional and averaged structur$\mathrm{al} /$ acoustical expression is

$$
\begin{aligned}
& \varphi p_{o}+\kappa_{a}\left[\operatorname{tr} \overline{\left[C_{i i}\right]^{-1}} \overrightarrow{\boldsymbol{I}}_{p} p_{o}-\operatorname{tr} \overline{\left[C_{i i}\right]^{-1} C_{i b}} \varphi \nabla_{x} \overline{\boldsymbol{u}_{o}}\right]+\kappa_{a} \varphi \nabla_{x} \cdot \overline{\boldsymbol{u}_{o}} \\
& +\kappa_{a} \nabla_{x} \cdot \overline{\boldsymbol{w}(\boldsymbol{x}, \boldsymbol{y})}=0 \text { in } D_{f}
\end{aligned}
$$




$$
\begin{aligned}
& p_{o}=\frac{-\kappa_{a}}{\left[\varphi+\kappa_{a} t r \overline{\left[C_{i i}\right]^{-1} \vec{I}}\right]}\left[-\operatorname{tr} \overline{\left[C_{i i}\right]^{-1} C_{i b}} \varphi \nabla_{x} \overline{\boldsymbol{u}_{o}}+\varphi \nabla_{x} \cdot \overline{\boldsymbol{u}_{o}}+\nabla_{x} \cdot \overline{\boldsymbol{w}(\boldsymbol{x}, \boldsymbol{y})}\right] \\
& p_{o}=-M\left[-\operatorname{tr} \overline{\left[C_{i i}\right]^{-1} C_{i b}} \varphi \nabla_{x} \overline{\boldsymbol{u}_{o}}+\varphi \nabla_{x} \cdot \overline{\boldsymbol{u}_{o}}+\nabla_{x} \cdot \overline{\boldsymbol{w}(\boldsymbol{x}, \boldsymbol{y})}\right]
\end{aligned}
$$

where

$$
M=\frac{\kappa_{a}}{\left[\varphi+\kappa_{a} t r{\overline{\left[C_{i i}\right.}}^{-1} \overrightarrow{\boldsymbol{I}}\right]}
$$

The grouping of Equations (40), (45), (44) and (50) develops one form of the coupled equation

$$
\begin{gathered}
-\omega^{2}\left(\bar{\rho} \boldsymbol{u}_{o}(\boldsymbol{x})+\rho_{o} \overline{\boldsymbol{w}(\boldsymbol{x}, \boldsymbol{y})}\right)=\nabla_{x} \cdot \overrightarrow{\overline{\boldsymbol{\tau}_{o}}}-\varphi \nabla_{x} p_{o}=\nabla_{x} \cdot\left(\overrightarrow{\overline{\boldsymbol{\tau}_{o}}}-\varphi p_{o} \overrightarrow{\boldsymbol{I}}\right) \\
-\omega^{2}\left(\varphi \rho_{f} \boldsymbol{u}_{o}(\boldsymbol{x})+\alpha(\omega) \varphi \rho_{f} \overline{\boldsymbol{w}(\boldsymbol{x}, \boldsymbol{y})}\right)=-\nabla_{x} p_{o} \\
\overrightarrow{\boldsymbol{\tau}_{o}}=\left[\overline{-C_{b i}\left[C_{i i}\right]^{-1} C_{i b}-C_{b b}}\right] \nabla_{x} \boldsymbol{u}_{o}+\overline{C_{b i}\left[C_{i i}\right]^{-1}} \overrightarrow{\boldsymbol{I}} p_{o} \\
\left.p_{o}=-\operatorname{Mtr} \overline{\left[C_{i i}\right]^{-1} C_{i b}} \varphi+\varphi\right] \nabla_{x} \overline{\boldsymbol{u}_{o}}-M \nabla_{x} \cdot \overline{\boldsymbol{w}(\boldsymbol{x}, \boldsymbol{y})}
\end{gathered}
$$

The term $\varphi p_{o} \vec{I}$ and the expression for $p_{o}$ will be subtracted from Equation (54)

$$
\begin{aligned}
& \overrightarrow{\boldsymbol{\tau}_{o}}-\varphi p_{o} \vec{I} \\
& =\left[\overline{-C_{b i}\left[C_{i i}\right]^{-1} C_{i b}-C_{b b}}+\left(\varphi \vec{I}-\overline{C_{b i}\left[C_{i i}\right]} \vec{I}_{p}\right) \operatorname{Mtr}\left(\varphi \vec{I}-\overline{\left[C_{i i}\right]^{-1} C_{i b}}\right)\right] \nabla_{x} \boldsymbol{u}_{o} \\
& +\left(\varphi \vec{I}-\overline{C_{b i}\left[C_{i i}{ }^{-1}\right.} \vec{I}_{p}\right) M \nabla_{x} \cdot \overline{\boldsymbol{w}(\boldsymbol{x}, \boldsymbol{y})}
\end{aligned}
$$

Equation (56) in its current form can model anisotropic material. A simpler expression can be obtained by assuming that the material is statistically isotropic. Therefore the first term in the right hand side can be expressed in the following form after taking advantage of basic concepts of symmetric tensors

$$
\left[-C_{b i}\left[C_{i i}\right]^{-1} C_{i b}-C_{b b}\right] \nabla_{x} \boldsymbol{u}_{o}=\lambda \nabla_{x} \cdot \boldsymbol{u}_{o} \vec{I}+\hat{\mu}\left[\nabla_{x} \boldsymbol{u}_{o}+\left[\nabla_{x} \boldsymbol{u}_{o}\right]^{T}\right]
$$

Additionally, in [6] it is shown that

$$
\overline{C_{b i}\left[C_{i i}\right]^{-1}} \overrightarrow{\boldsymbol{I}_{p}}=\operatorname{tr} \overline{\left[C_{i i}\right]^{-1} C_{i b}}
$$

Set $\operatorname{tr}\left(\varphi \overrightarrow{\boldsymbol{I}}+\overline{\left[C_{i i}\right]^{-1} C_{i b}}\right) \nabla_{x} \boldsymbol{u}_{o}=\beta \nabla_{x} \cdot \boldsymbol{u}_{o}$ and because of (58).

$$
\beta=\left(\varphi+\frac{1}{3} \operatorname{tr} \overline{C_{b i}\left[C_{i i}\right]^{-1}} \overrightarrow{\boldsymbol{I}}_{p}\right) \text {. Analyzing how Equation (58) was derived, } \beta
$$

represents the proportion of fluid pressure that produces the same strains as the total stress.

Plugging Equation (57) and the definition for $\beta$ into Equation (56) the subsequent equation is obtained 


$$
\begin{aligned}
& \overrightarrow{\tau_{o}}-\varphi p_{o} \vec{I} \\
& =\lambda \nabla_{x} \cdot \boldsymbol{u}_{o} \vec{I}+\hat{\mu}\left[\nabla_{x} \boldsymbol{u}_{o}+\left[\nabla_{x} \boldsymbol{u}_{o}\right]^{T}\right]+\beta^{2} M \nabla_{x} \cdot \boldsymbol{u}_{o} \vec{I}+\beta M \nabla_{x} \cdot \overline{\boldsymbol{w}(\boldsymbol{x}, \boldsymbol{y})} \overrightarrow{\boldsymbol{I}} \\
& =\left(\lambda+\beta^{2} M\right) \nabla_{x} \cdot \boldsymbol{u}_{o} \vec{I}+\hat{\mu}\left[\nabla_{x} \boldsymbol{u}_{o}+\left[\nabla_{x} \boldsymbol{u}_{o}\right]^{T}\right]+\beta M \nabla_{x} \overline{\boldsymbol{w}(\boldsymbol{x}, \boldsymbol{y})} \overrightarrow{\boldsymbol{I}}
\end{aligned}
$$

$\lambda$ and $\hat{\mu}$ are new Lame' constants of the elastic portion when the porous material is drained where as $\left(\lambda+\beta^{2} M\right)$ is the contained Lame' constant sometimes referred as $\lambda_{c}$ in the literature. Equation (59) is in the exact form as Equation (30) found in Biot and Willis' paper [10]. Equation (55) can also be expressed, using the definition for $\beta$, as

$$
p_{o}=-\beta M \nabla_{x} \cdot \overline{\boldsymbol{u}_{o}}-M \nabla_{x} \cdot \overline{\boldsymbol{w}(\boldsymbol{x}, \boldsymbol{y})}
$$

It is desired to define the equations above as a function of $\boldsymbol{u}_{o}$ and $U_{o}$. Hence, the relative displacement can be expressed as $\overline{\boldsymbol{w}(\boldsymbol{x}, \boldsymbol{y})}=\left[\overline{\boldsymbol{U}_{o}}-\varphi \overline{\boldsymbol{u}_{o}}\right]$. Equation (53) becomes

$$
\begin{array}{r}
-\omega^{2}\left(\rho_{f} \boldsymbol{u}_{o}(\boldsymbol{x})+\alpha(\omega) \varphi \rho_{f}\left(\overline{\boldsymbol{U}_{o}}-\varphi \overline{\boldsymbol{u}_{o}}\right)\right)=-\nabla_{x} p_{o} \\
-\omega^{2}\left(\left(\varphi \rho_{f}-\alpha(\omega) \varphi^{2} \rho_{f}\right) \boldsymbol{u}_{o}(\boldsymbol{x})+\alpha(\omega) \varphi \rho_{f} \overline{\boldsymbol{U}_{o}}\right)=-\nabla_{x} p_{o}
\end{array}
$$

Now, subtracting (61) from (52) one obtains

$$
\left.-\omega^{2}\left((1-\varphi) \rho_{s}+\alpha(\omega) \varphi^{2} \rho_{f}-\varphi \rho_{f}\right) \boldsymbol{u}_{o}(\boldsymbol{x})+\left(\rho_{o}-\alpha(\omega) \varphi \rho_{f}\right) \overline{\boldsymbol{U}_{o}}\right)=\nabla_{x} \cdot \overrightarrow{\overline{\tau_{o}}}
$$

Applying the same definition of $i \omega \overline{\boldsymbol{w}(\boldsymbol{x}, \boldsymbol{y})}$ into Equation (53) the acoustic portion of the coupled equation of motion turns into

$$
\begin{aligned}
& -\omega^{2}\left(\varphi \rho_{f} \boldsymbol{u}_{o}(\boldsymbol{x})+\alpha(\omega) \varphi \rho_{f}\left(\varphi \overline{\boldsymbol{U}_{o}}-\varphi \overline{\boldsymbol{u}_{o}}\right)\right) \\
& =-\omega^{2}\left(\left(\varphi \rho_{f}-\alpha(\omega) \varphi^{2} \rho_{f}\right) \overline{\boldsymbol{u}_{o}}+\alpha(\omega) \varphi^{2} \rho_{f} \overline{\boldsymbol{U}_{o}}\right)=-\nabla_{x} p_{o}
\end{aligned}
$$

Set

$$
\begin{gathered}
\widetilde{\rho_{11}}=(1-\varphi) \rho_{s}+\alpha(\omega) \varphi^{2} \rho_{f}-\varphi \rho_{f} \\
\widetilde{\rho_{12}}=\rho_{f}-\alpha(\omega) \varphi \rho_{f} \\
\widetilde{\rho_{22}}=\alpha(\omega) \varphi \rho_{f}
\end{gathered}
$$

so that (63) and (64) can be expressed in a simpler form

$$
\begin{gathered}
-\omega^{2}\left(\widetilde{\rho_{11}} \overline{\boldsymbol{u}_{o}}(\boldsymbol{x})+\widetilde{\rho_{12}} \overline{\boldsymbol{U}_{o}}\right)=\nabla_{x} \cdot \overline{\overline{\tau_{o}}} \\
-\omega^{2}\left(\widetilde{\rho_{12}} \overline{\boldsymbol{u}_{o}}+\widetilde{\rho_{22}} \overline{\boldsymbol{U}_{o}}\right)=-\nabla_{x} p_{o}
\end{gathered}
$$

Plug into (68) and (69) the definitions for $\overline{\overline{\tau_{o}}}$ and $p_{o}$ found in Equations (54) and (55), along with the replacements for $\beta$ and $\overline{\boldsymbol{w}(\boldsymbol{x}, \boldsymbol{y})}$ one arrives to the following equation

$$
\begin{aligned}
&-\omega^{2}\left(\widetilde{\rho_{11}} \overline{\boldsymbol{u}_{o}}(\boldsymbol{x})+\widetilde{\rho_{12}} \overline{\boldsymbol{U}_{o}}\right) \\
&=\left(\lambda+M(\beta-\varphi)^{2}\right) \nabla_{x} \nabla_{x} \cdot \overline{\boldsymbol{u}_{o}}+(\beta-\varphi) M \nabla_{x} \nabla_{x} \cdot \overline{\boldsymbol{U}_{o}}+\hat{\mu} \nabla_{x}^{2} \overline{\boldsymbol{u}_{o}} \\
&-\omega^{2}\left(\widetilde{\rho_{11}} \overline{\boldsymbol{u}_{o}}(\boldsymbol{x})+\widetilde{\rho_{12}} \overline{\boldsymbol{U}_{o}}\right)=(P-2 N) \nabla_{x} \nabla_{x} \cdot \overline{\boldsymbol{u}_{o}}+Q \nabla_{x} \nabla_{x} \cdot \overline{\boldsymbol{U}_{o}}+2 N \nabla_{x}^{2} \overline{\boldsymbol{u}_{o}}
\end{aligned}
$$


and

$$
\begin{gathered}
-\omega^{2}\left(\widetilde{\rho_{12}} \overline{\boldsymbol{u}_{o}}+\widetilde{\rho_{22}} \overline{\boldsymbol{U}_{o}}\right)=\varphi^{2} M \nabla_{x} \nabla_{x} \cdot \overline{\boldsymbol{U}_{o}}+(\beta-\varphi) M \nabla_{x} \nabla_{x} \cdot \overline{\boldsymbol{u}_{o}} \\
-\omega^{2}\left(\widetilde{\rho_{12}} \overline{\boldsymbol{u}_{o}}+\widetilde{\rho_{22}} \overline{\boldsymbol{U}_{o}}\right)=R \nabla_{x} \nabla_{x} \cdot \overline{\boldsymbol{U}_{o}}+Q \nabla_{x} \nabla_{x} \cdot \overline{\boldsymbol{u}_{o}}
\end{gathered}
$$

Equation 70(a) and Equation 70(b) and Equation 71(a) and Equation 71(b) are the Equation which will be utilized to study wave propagation through porous medium. Note, that the form of the equations are in the same format as $\mathrm{Bi}$ ot's Equation [11]

\section{Results and Conclusion}

A Simple application of Equations (70) and (71) is applied to the simple layer configuration shown in Figure 1. The figure shows a multi layer system where there is a plate that is glued to foam 1 . The parameters pertaining to plate 1 and foam 1 are listed in Table 1. This simulation also applied the following parameters that are not listed in the table: Viscous length $=40 \mu \mathrm{m}$, Thermal length $=80$ $\square \mathrm{m}$. The goal of this simulation is to calculate Random Incidence Transmission Loss (TL). This is achieved by applying Finite Size correction Transfer Matrix Method (FTMM). The Finite Size Correction is achieved by incorporating a Green's Function integration to the radiation efficiency. Equations (70) and (71) are used to simulate how the structural and fluid stresses will change as the acoustic wave travels through the panel. The results are shown in Figure 2. The results are compared to measured results and also to the empirical formulas of Delaney and Bazley [12] [13]. The graph shows that the calculated results come within $0.4 \mathrm{~dB}$.

\begin{tabular}{|l|c|c|c|c|}
\hline Case4 & Air & Plate1_B* & Foam1 & Air \\
\hline Case43 & Air & Foam1_B & Plate1* & Air \\
\hline
\end{tabular}

Figure 1. Multi layer cofiguration.

\begin{tabular}{|c|c|c|c|c|c|c|c|c|c|c|c|c|}
\hline Panel & Thickness [m] & \multicolumn{2}{|c|}{ Density $\left[\mathrm{kg} / \mathrm{m}^{3}\right]$} & \multicolumn{2}{|c|}{ Youngs Modulus [Pa] } & \multicolumn{3}{|c|}{ Shear Modulus $[\mathrm{Pa}]$} & \multicolumn{2}{|c|}{ Poisson Ratio } & Loss Factor & Description \\
\hline Plate1 & $7.00 \mathrm{E}-04$ & 7800 & & $2.10 \mathrm{E}+11$ & & 8.00 & +10 & & 0.3125 & & 0.001 & Steel \\
\hline Plate2 & $1.00 \mathrm{E}-03$ & 1100 & & $2.30 \mathrm{E}+09$ & & 7.72 & +08 & & 0.4896 & & 0.005 & Hard Rubber \\
\hline Plate3 & $2.10 \mathrm{E}-03$ & 2500 & & $4.85 \mathrm{E}+10$ & & 1.96 & +10 & & 0.2398 & & 0.001 & Tempered Glass \\
\hline Foam & Thickness [m] & $\begin{array}{l}\text { Density } \\
{\left[\mathrm{kg} / \mathrm{m}^{3}\right]}\end{array}$ & $\begin{array}{l}\text { You } \\
\text { Mod }\end{array}$ & $\begin{array}{l}\text { lg's } \\
\text { ulus [Pa] }\end{array}$ & $\begin{array}{l}\text { Poisson } \\
\text { Ratio }\end{array}$ & $\begin{array}{l}\text { Loss } \\
\text { Factor }\end{array}$ & Porosity & & rtuosity & $\begin{array}{l}\text { Flow } \\
\text { Resis }\end{array}$ & tivity $\left[\mathrm{Ns} / \mathrm{m}^{4}\right]$ & Description \\
\hline Foam 1 & $1.00 \mathrm{E}-02$ & 22 & 4.65 & +04 & 0.4 & 0.14 & 0.96 & 1.7 & & 5000 & & $\begin{array}{l}\text { Polyurethane } \\
\text { Foam }\end{array}$ \\
\hline Foam 2 & $3.00 \mathrm{E}-02$ & 40 & 9.50 & +04 & 0.34 & 0.1 & 0.95 & 1.9 & & $1.15 \mathrm{H}$ & +04 & $\begin{array}{l}\text { Typical Car } \\
\text { Seat Foam }\end{array}$ \\
\hline
\end{tabular}

Table 1. Parameters pertaining to plate and foam. 

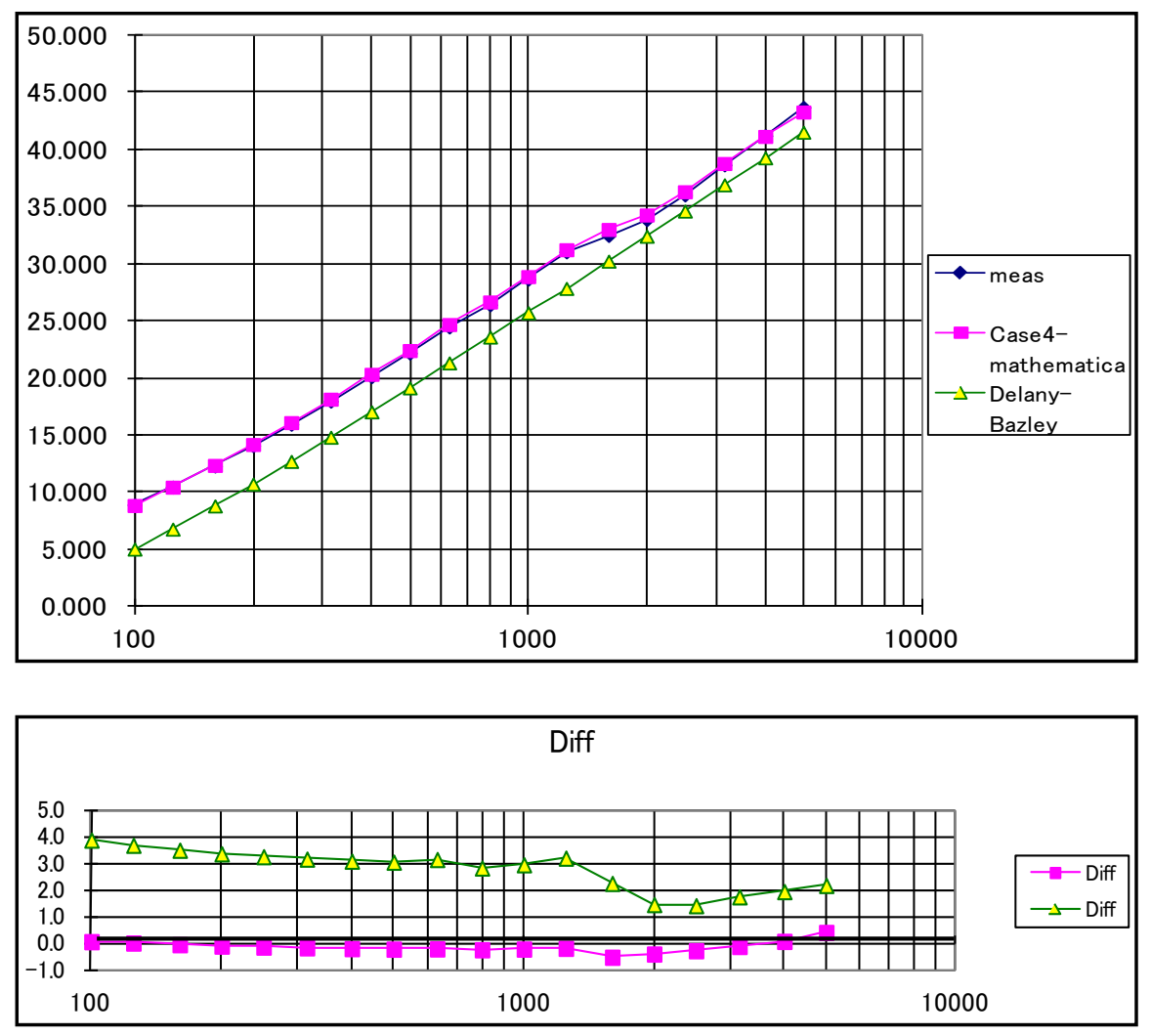

Figure 2. Top: TL comparison $(\mathrm{dB})$; Bottom: TL difference $\mathrm{c}(\mathrm{dB})$ measured data is the reference.

The current method used in order to calculate TL requires the use of various measured variables. Parameters like tortuosity, viscous/thermal lengths, tortuosity, flow resistivity are all measured. Additionally, these measurements are being done at different times at different facilities that have disparate techniques and equipment. This makes running simulations challenging. One of the goals of going through this mathematical derivation is to find systematic techniques to reduce the disparity in these parameters or at least get a better understanding of the physics.

\section{Conflicts of Interest}

The authors declare no conflicts of interest regarding the publication of this paper.

\section{References}

[1] Lyon, R. and DeJong, R. (1990) Theory and Application of Statistical Energy Analysis. 2nd Edition, Butterworth-Heinemann.

[2] Langley, R. and Bremmer, P. (1999) A Hybrid Method for the Vibration Analysis of Complex Structural-Acoustic Systems. J. Acoust. Soc. Am., 105, 1657-1671. https://doi.org/10.1121/1.426705

[3] Teagle, A., Ichikawa, S., Matsuda, T. and Ide, F. (2019) PDT Application: Systematic Decision Tool for Seeking Optimum NV Performance. Proceeding SAE WCX 
World Congress, Detroit, 5-19. (In Press)

[4] Sanchez-Palencia, E. (1980) Non-Homogenous Media and Vibration Theory. Lecture Notes in Physics 127, Springer-Verlag, New York.

[5] Burridge, R. and Keller, J. (1981) Poroelasticity Equations Derived from Microstructure. J. Acoust. Soc. Am., 70, 1140-1146. https://doi.org/10.1121/1.386945

[6] Bensoussan, A., Lions, L. and Papanicolaou, G. (1978) Asymptotic Analysis for Periodic Structures. Studies in Mathematics and its Application, 5, North-Holland, Amsterdam.

[7] Zwikker, C. and Kosten, C. (1949) Sound Absorbing Materials. Elsevier, New York.

[8] Johnson, D. and Koplik, J. (1987) Theory of Dynamic Permeability and Totuosity in Fluid-Saturated Porous Media. Journal of Fluid Mechanics, 176, 379-402. https://doi.org/10.1017/S0022112087000727

[9] Landau, L. and Lifshitz, E. (1959) Fluid Mechanics. Pergamon.

[10] Biot, M. and Willis, D. (1957) The Elastic Coefficient of the Theory of Consolidation. Journal of Applied Mechanics, 24, 594-601.

[11] Biot, M. (1962) Mechanics of Deformation and Acoustic Propagation in Porous Media. Journal of Applied Physics, 34, 1482-1498. https://doi.org/10.1063/1.1728759

[12] Delany, M. and Bazley, E. (1970) Acoustical Properties of Fibrous Materials. Applied Acoustics, 3, 105-116. https://doi.org/10.1016/0003-682X(70)90031-9

[13] Miki, Y. (1990) Acoustical Properties of Porous Materials-Modification of Delany-Bazley Models. Journal of the Acoustical Society of Japan, 11, 19-24. https://doi.org/10.1250/ast.11.19 


\section{Nomenclature}

$t r=$ trace of a tensor

$\bar{a}=$ averaged at the microscopic level

$v=$ Fluid Velocity

$u=$ Structural displacement

$U=$ Fluid displacement

$p=$ Fluid Pressure

$\varphi=$ Porosity

$\eta=$ Viscosity

$\xi=$ Second Viscosity

$D_{f}=$ Domain Occupied by Fluid

$D_{s}=$ Domain Occupied by Structure

$\partial D_{f}=$ Boundary of Domain Occupied by Fluid

$\partial D_{s}=$ Boundary of Domain Occupied by Structure

$\gamma=C_{p} / C_{V} C_{p}=$ specific heat at constant pressure $\mathrm{C}_{\mathrm{v}}=$ Specific heat at constant volume

$\kappa=$ Coefficient of thermal Conductivity

$\operatorname{Pr}=$ Prandlt Number $=\frac{\eta C_{p}}{\kappa}$

$n=$ unit normal pointing into the solid

$\vec{\sigma}=$ Stress Tensor in the Fluid

$\vec{\tau}=$ Stress Tensor in the structure

$\widehat{C^{i j k l}}=$ Elastic fourth ranked Contravariant tensor, operates on $\nabla u$

$\rho_{f}=$ density of the Fluid

$\rho_{s}=$ density of the Structure

$\lambda, \hat{\mu}=$ Lame Parameters

$\kappa=$ Coefficient of thermal Conductivity

$T=$ Temperature deviation

$k(\omega)=$ Dynamic Viscous Permeability

$k^{\prime}(\omega)=$ Dynamic Thermal Permeability

$\kappa_{a}=$ Acoustic bulk Modulus

$a^{*}=$ asterisk superscript means scaled variable (dimensionless) 